\title{
BRUNO POTTIER
}

\section{LES CIRCONCELLIONS}

\author{
Formation d'une élite rurale monastique autonome \\ dans l'Afrique du Nord des $I V^{e}$ et $V^{e}$ siècles
}

Les circoncellions constituent un groupe social dans l'Afrique chrétienne des $\mathrm{IV}^{\mathrm{e}}$ et $\mathrm{V}^{\mathrm{e}}$ siècles qui a été l'objet de nombreuses interprétations. Leur étude a été en effet obscurcie par des enjeux théoriques, portant sur la capacité de mouvements religieux de comporter des aspects sociaux. Les circoncellions ont trouvé leur terreau dans le schisme donatiste, qui est né de la protestation d'une partie des chrétiens d'Afrique du Nord, et surtout de Numidie, devant la réintégration dans l'Église des lapsi, les chrétiens ayant sacrifié aux dieux païens lors de la persécution de Dioclétien entre 303 et 305. Les circoncellions donatistes ont mené des actions contre des propriétaires terriens en Numidie dans la première moitié du IV siècle. Ils ont défendu vers 347 l'évêque Donatus de Bagaï contre les commissaires impériaux Macaire et Paul envoyés par l'empereur Constant unifier les deux Églises. Les circoncellions sont réapparus à l'extrême fin $d u \mathrm{IV}^{\mathrm{e}}$ siècle et au début $\mathrm{du} \mathrm{V}^{\mathrm{e}}$ siècle dans les cuvres de saint Augustin, qui les a accusés de commettre de nombreuses attaques contre des prêtres catholiques, surtout après l'interdiction définitive du donatisme en 405 .

Les circoncellions ont tout d'abord été perçus soit comme de simples bandits, soit comme des révolutionnaires sociaux ${ }^{1}$. Suivant l'opinion de Charles Saumagne, qui reste communément admise, ils auraient constitué des groupes de travailleurs agricoles ${ }^{2}$. Inspiré par la période de la décolonisation, William Frend a tenté en revanche de prouver que le donatisme aurait été un mouvement de protestation berbère contre la présence romaine, dont les circoncellions auraient été l'aile agissante ${ }^{3}$. Cette interprétation repose comme celle de Jean-Paul Brisson sur la valorisation anachronique d'un nationalisme africain opposé à Rome. William Frend a de plus tenté d'identifier les circoncellions à

\footnotetext{
${ }^{1}$ Pour un bilan récent sur l'historiographie des circoncellions, voir John Michael GaDDIS, There is No Crime for Those Who Have Christ: Religious Violence in the Christian Roman Empire, Ph.D. Princeton University 1999, p. 88-98.

2 Charles SAUMAGNE, Ouvriers agricoles ou rôdeurs de celliers? Les circoncellions d'Afrique, dans: Annales E. S. C. 6 (1934), p. 355-364.

${ }^{3}$ William FREND, The Donatist Church: A Movement of Protest in Roman North Africa, Oxford 1952; Jean-Paul BRISSON, Autonomisme et christianisme dans l'Afrique romaine de Septime Sévère à l'invasion vandale, Paris 1958.
} 
des confréries itinérantes monastiques, une opinion qui a été aussi soutenue par Salvatore Calderone en utilisant des arguments différents ${ }^{4}$. Des historiens d'inspiration marxiste comme Hans Diesner, souvent originaires d'Allemagne de l'Est, ont insisté sur l'aspect social de l'hérésie donatiste, qui aurait constitué un mouvement révolutionnaire contre les propriétaires terriens ${ }^{5}$. Ces interprétations marxistes, qui défendent l'idée d'une unicité du mouvement des circoncellions aux $I V^{\mathrm{e}}$ et $\mathrm{V}^{\mathrm{e}}$ siècles, n'ont lui attribué aucune spécificité. Les circoncellions, identifiés à des groupes organisés de travailleurs agricoles, conformément à la lecture de Charles Saumagne, n'aurait été en effet que l'élément agissant, voire l'avant-garde, des populations rurales de Numidie. Emin Tengström, auteur d'une étude qui fait aujourd'hui référence, a tenté en partie de concilier ces diverses théories ${ }^{6}$. Selon lui, les circoncellions auraient constitué un ordo de travailleurs agricoles qui aurait participé à des violences contre des propriétaires terriens dans la première moitié du IV ${ }^{e}$ siècle. Certains membres de cet ordre auraient formé à la fin du $\mathrm{IV}^{\mathrm{e}}$ siècle un groupe fanatique donatiste. Emin Tengström a utilement montré la fragilité des thèses évoquant l'existence d'un mouvement révolutionnaire ou d'un mouvement national à cette époque en Afrique du Nord, qui reposent finalement sur une surinterprétation des sources. Cependant, ses travaux marquent deux tendances significatives, qui dénotent un parti pris actuel de la recherche sur ces questions. On observe en effet un refus implicite d'imaginer que des moines aient pu commettre des actes de violence, que l'on prefère attribuer à des fanatiques laïcs, et une tendance à séparer nettement, en distinguant leurs auteurs, violences religieuses et violences à caractère social ${ }^{7}$. Hans Diesner a suivi la même tendance en séparant des circoncellions proprement dits, formant un ordo de travailleurs agricoles, et des groupes de fanatiques religieux qui auraient été assimilés à tort à ces derniers. Zeev Rubin a établi dans une étude récente la même distinction entre mouvement religieux et troubles sociaux, mais de manière inversée. Il a séparé en effet les agonistici, le terme que les circoncellions utilisaient eux-mêmes pour se définir, qui auraient formé un mouvement monastique violemment opposé aux catholiques, des circoncellions propre-

${ }^{4}$ Salvatore CALDERone, Circumcelliones, dans: Parola del Passato, 22, 1967, p. 94-109; William FREND, The cellae of the African Circumcelliones, dans: Journal of Theological Studies n. s. 3 (1952), p. 87-89; Circumcelliones and Monks, dans: Journal of Theological Studies 20 (1969), p. 539-547.

${ }^{5}$ Hans DIESNER, Kirche und Staat im spătrömischen Reich: Aufsătze zur Spătantike und zur Geschichte der Alten Kirche, Berlin 1963, p. 53-78.

${ }^{6} \mathrm{Cf}$. Emin TENGSTROM, Donatisten und Katholiken. Soziale, wirtschaftliche und politische Aspekte einer nordafrikanischen Kirchenspaltung, Goteborg 1964, p. 70-71. Voir aussi Y. Congar, dans son introduction aux: Euvres de saint Augustin, $t$. 28: Traités anti-donatistes, Paris 1963, p. 32-37.

${ }^{7}$ Cette tendance a été initiée par Albert JONES, Were Ancient Heresies National or Social Movements in Disguise?, dans: Journal of Theological Studies 10/2 (1959), p. 280-298. 
ment dits, un terme qui aurait désigné de façon péjorative des bandits pillant des domaines ruraux en Numidie dans la première moitié du $\Gamma V^{e}$ siècle ${ }^{8}$. Ainsi, plusieurs études actuelles proposent des jugements proches de celui de Frédéric Martroye et de Paul Monceaux au début du $\mathrm{XX}^{\mathrm{e}}$ siècle, assimilant les circoncellions à des bandits, à la suite d'Optat de Milev et d'Augustin, et à des fanatiques, un terme qui n'est pas sans compter de fortes résonances contemporaines. La juste appréciation des déformations que les auteurs catholiques ont fait subir au récit des événements à des fins polémiques constitue d'ailleurs l'un des principaux enjeux de toute étude sur les circoncellions. Je me propose ainsi de remettre dans son contexte le phénomène des circoncellions qui a été trop souvent étudié indépendamment des cadres sociaux et culturels de l'Afrique des $I V^{e}$ et $V^{e}$ siècles.

L'interprétation de Salvatore Calderone, que nous allons renforcer par de nouveaux arguments, se démarque radicalement de celle de Charles Saumagne, car elle assimile les circoncellions à des groupes de moines itinérants ${ }^{9}$. Le principal argument mis en avant par William Frend à l'appui de la même hypothèse, reposant uniquement sur des considérations étymologiques, est plus fragile $^{10}$. Salvatore Calderone a remarqué l'insistance de saint Augustin à décrire les circoncellions comme de faux moines vagabonds, significativement dans deux sermons destinés aux catholiques, datant respectivement de 401 et de 407 ou 412. Augustin oppose dans le premier sermon les moines égyptiens aux circoncellions ${ }^{11}$, puis dans le second, à l'occasion d'un commentaire du psaume 132, plus généralement les moines catholiques, qui seraient tous assemblés en communautés, à ces mêmes circoncellions ${ }^{12}$. Augustin, après l'interdiction officielle du donatisme en 405 , a ainsi éprouvé le besoin de convaincre ses propres fidèles qu'ils ne devaient pas respecter en tant que moines les cir-

${ }^{8}$ Cette analyse a été contestée à juste titre par V. Neri, qui défend la possibilité de mouvements liant préoccupations sociales et religieuses, cet auteur évoquant néanmoins dans son étude uniquement l'époque d'Augustin. Cf. Zvin RuBIN, Mass Movements in Late Antiquity, dans: Leaders and Masses in the Roman World. Studies in honor of Z. Yavetz, Leyde, New York, Cologne 1995, p. 156-179; Valerio NERI, I Marginali nell'Occidente Tardoantico. Poveri, Infames e Criminali nella nascente società cristiana, Bari 1998, p. 168-177, spécialement p. 168 , n. 160.

${ }^{9}$ Cette interprétation a été reprise récemment par David Caner, sans qu'il éprouve le besoin de la démontrer. Cf. David CANER, Wandering, Begging Monks. Spiritual Authority and the Promotion of Monaticism in Late Antiquity, Berkeley, Los Angeles, London 2002, p. 230-233.

${ }^{10}$ Selon William Frend, le terme circoncellions aurait fait référence aux moines effectuant un circuit entre les diverses cellae des martyres, plutôt qu'à des personnes rodant autours des celliers.

${ }^{11}$ Ep. ad Cath., XVI, 40 (401).

${ }^{12}$ En. in Ps., 132, 3 (discours ad populum tenu à Hippone le 8 avril 407 ou à Carthage le 23 décembre 412): Ex voc huius psalmi appellati sunt et monachi, ne quis vobis de isto nomine insultet catholicis, quando vos recte haereticis de circumcellionibus insultare coeperitis, ut erubescendo salventur, illi vobis insultant de monachis. 
concellions. La lutte des circoncellions contre le paganisme dans les années 399-400 a sûrement renforcé leur prestige auprès des catholiques. Augustin dans un discours aux catholiques d'Hippone ou de Carthage sans doute daté de 399 leur a déconseillé en effet de détruire eux-mêmes les idoles païennes, comme le faisaient les circoncellions ${ }^{13}$. De plus, les critiques d'Augustin contre les relations sexuelles supposées existant entre circoncellions et sanctimoniales donatistes, une sorte de lapsus involontaire sur le caractère monastique de femmes donatistes, ne peuvent avoir eu comme objectif que de contredire la réputation de chasteté de ceux- $\mathrm{ci}^{14}$. Le témoignage de Possidius de Calama, un évêque catholique auteur d'une biographie d'Augustin dans les années 430 lui-même menacé par les circoncellions, est particulièrement important. Il attribue à des circoncellions errants supposés mettre en avant leur chasteté, sub professione continentium, tant les troubles de l'époque du comte Taurinus dans la première moitié du $I^{\mathrm{e}}$ siècle que les attaques de prêtres catholiques du début du $V^{e}$ siècle $^{15}$. Très clairement en effet, Possidius isole deux phases successives d'action des mêmes groupes de moines itinérants pratiquant la chasteté, successivement marquées par des préoccupations principalement sociales puis religieuses. Possidius oppose d'ailleurs explicitement les circoncellions se faisant passer pour des moines aux continents et aux vierges du monastère fondé par saint Augustin à Hippone, qui étaient soumis à la stricte autorité de leur évêque ${ }^{16}$. Le seul critère permettant de définir un moine était l'exercice de la chasteté à une époque où les communautés ordonnées étaient encore minoritaires. L'identification des circoncellions à des moines, au moins pour le début du $V^{\mathfrak{e}}$ siècle, semble donc peu contestable. Les moines gyrovagues ne constituent donc pas une spécificité de la partie orientale de l'Empire, Syrie et Égypte. Un argument nouveau, concernant le devenir des circoncellions après les interdictions répétées du donatisme en 405 et surtout en 411 , renforce encore cette hypothèse.

Il n'est pas étonnant, qu'à partir de la conférence de Carthage de 411 interdisant définitivement le donatisme, les mesures qui ont été prises contre les circoncellions aient pour objectif d'empêcher leur itinérance. Un édit du tribun Marcellinus, promulgué à cette occasion, a imposé aux possessores de réprimer l'insolentia des circoncellions troublant la quies publica qu'ils savaient être présents sur leur domaine, sous peine de voir celui-ci confisqué ${ }^{17}$. Saint Augustin, dans le Contra Gaudentium, écrit entre 419 et 422 , a affirmé que la

\footnotetext{
${ }^{13}$ Sermo 62, 10, 17.

${ }^{14}$ C. Ep. Parm., II, 9, 19; C. Gaud., I, 36, 46.

${ }^{15}$ Possidius, Vita Aug., X: Habebant etiam iidem Donatistae per suas pene omnes Ecclesias inauditum hominum genus perversum ac violentum, velut sub professione continentium ambulantes, qui Circumcelliones dicebantur.

${ }^{16}$ Ibid., XI.

${ }^{17}$ Edictum, 1. 80-83 (SC, 411, p. 974-975).
} 
majorité des circoncellions, qui respectaient désormais la discipline de l'Église catholique et avaient été contraints à cultiver les champs, pratiquaient la chasteté $^{18}$. Ils avaient donc cessé d'être des vagi, des errants et des paresseux refusant les travaux des champs, à la satisfaction d'Augustin. Les circoncellions ont ainsi continué de vivre comme des moines, en gardant ce qui faisait leur spécificité, l'abstinence sexuelle, pour marquer leur différence par rapport aux autres paysans. Augustin rapporte l'existence de l'hérésie des Abeloim, professée par les habitants d'un village du territoire d'Hippone s'astreignant à une parfaite chasteté ${ }^{19}$. Il a donc existé de nombreux courants ascétiques au sein des populations rurales de Numidie au $I V^{e}$ et $V^{e}$ siècles, les circoncellions ne formant que l'un d'entre eux, en lien avec une exceptionnelle christianisation des campagnes. Ce passage du Contra Gaudentium s'explique par l'application d'une loi célèbre de Gratien de 382 imposant que les mendiants valides de naissance libre, auxquels pouvaient être assimilés les circoncellions, soient affectés comme colons à des propriétaires terriens ${ }^{20}$. Ainsi, la plupart des circoncellions ont effectivement disparu après 411 et la conférence de Carthage, parce qu'ils ne pouvaient exister qu'en obtenant une certaine reconnaissance par l'État. Dans l'alternative contraire, plus rien ne protégeait alors ces derniers de la politique cohérente suivie par divers empereurs du IV $V^{e}$ siècle contre les populations itinérantes, vagi et mendiants.

Cette constatation permet de limiter le poids du principal argument utilisé par Charles Saumagne et Emin Tengström pour identifier les circoncellions à des travailleurs agricoles. Ils se sont principalement basés sur une loi d'Honorius de 412 dans laquelle étaient fixées des amendes proportionnelles aux membres donatistes des divers ordines, décurions, marchands, plébéiens et circoncellions $^{21}$. Or, l'identification des circoncellions à des travailleurs agricoles repose sur les hasards de la documentation épigraphique. Sans la découverte providentielle de l'inscription de Mactar, évoquant un moissonneur devenu chef d'équipe de travailleurs agricoles puis notable municipal au III $^{\mathrm{e}}$ siècle, cette identification n'aurait sans doute jamais été évoquée ${ }^{22}$. D'autre part, supposant une certaine permanence des relations sociales dans l'Afrique du Nord, Charles Saumagne a rapproché des circoncellions les équipes de travailleurs agricoles se louant sur les marchés qu'il pouvait observer dans l'Algérie coloniale du début du $\mathrm{XX}^{\mathrm{e}}$ siècle. Or, cette interprétation semble être insuffisamment fondée. Un groupe social se voyait reconnu par l'État romain

\footnotetext{
${ }_{18}$ Augustin, C. Gaud., I, 29, 33: Neque enim isti, qui pereunt, illorum saltem numero aequantur, qui ex ipso genere nunc iam tenentur ordine disciplinae colendisque agris amisso circumcellionum et opere et nomine inseruiunt, servant castitatem, tenent unitatem.

${ }^{19}$ Augustin, De haeresibus, 87.

${ }^{20} \mathrm{CTh} ., \mathrm{XIV}, 18,1$ (De mendicantibus non invalidis).

${ }^{21}$ CTh., XVI, 5, 52 .

${ }^{22}$ CIL VIII, 11824.
} 
un statut d'ordo uniquement s'il remplissait pour celui-ci une mission particulière, souvent sous la forme d'une obligation fiscale ou administrative spécifique $^{23}$. Ainsi les negotiatores, soumis au paiement de la collatio lustralis, impôt urbain, formaient bien un ordre. Les travailleurs agricoles itinérants ne pouvaient donc former une catégorie fiscale autonome et un ordo, étant enregistrés soit sous le nom d'un propriétaire terrien s'ils résidaient en permanence sur son domaine, par exemple s'ils ne possédaient aucune terre, soit sous leur propre nom dans leur lieu de résidence s'ils étaient aussi de petits propriétaires terriens, comme le moissonneur de Mactar. On ne peut comprendre d'autre part pourquoi Honorius aurait séparé le traitement des travailleurs agricoles de celui des plebei, en demandant aux circoncellions une amende sept fois inférieure à ces derniers. Emin Tengström partage ainsi avec les historiens marxistes qu'il a critiqué une image fausse des circoncellions comme une sorte d'aristocratie ouvrière, disposant de revenus élevés, agissant comme une »avant-garde du prolétariat« rural.

La loi d'Honorius de 412 semble plutôt citer les circoncellions comme formant un ordo de moines, dont la pauvreté et la mendicité serait reconnue comme principale caractéristique. En effet, les moines, au contraire des travailleurs agricoles, formaient bien un ordo puisque l'État les reconnaissait, à l'égal des clercs, comme formant une catégorie sociale utile à l'État par leur piété. Une loi de Constance II de 361 accorde aux personnes qui, dans les villes et les villages, ont fait preuve de leur vertu à la suite d'un vœu, bien évidemment des moines, d'être déchargés de toute obligation fiscale. Une loi de Valentinien de 368 dispense les vierges consacrées du paiement de la capitation $^{24}$. La loi de Constance II de 361 peut très bien faire allusion à des ascètes itinérants typiques du monachisme syrien de l'époque. En effet, il n'existait pas de congrégation monastique vers 360 , à l'exception des disciples de Pachôme en Égypte et de Basile de Césarée en Anatolie.

Il paraitrait très étonnant $a$ priori qu'Honorius reconnaisse en 412 les circoncellions hérétiques comme formant un ordo, quelques mois après la conférence de Carthage imposant en août 411 la réunion autoritaire des deux égli$\operatorname{ses}^{25}$. Cependant, la loi d'Honorius de 412 suppose que tous les circoncellions n'étaient pas donatistes. En effet, les circoncellions sont placés en parallèle avec les décurions, marchands et plébéiens. Seuls les membres donatistes de ces catégories devaient payer une amende. Or, il existait à cette époque en Afrique des moines itinérants catholiques. Augustin a en effet dénoncé très

\footnotetext{
${ }^{23}$ Pour les ordines de l'époque tardive, voir Jean-Michel CARRIE, Les associations professionnelles à l'époque tardive: entre munus et convivialité, dans: ID., Rita LIzZI (dir.), Humana sapit. Etudes d'Antiquité tardive offertes à Lellia Cracco-Ruggini, Paris 2002, p. 309332.

${ }^{24}$ CTh., XVI, 2, 16; XIII, 10, 4 .

${ }^{25}$ Selon Roland Delmaire, la datation de cette loi serait cependant à revoir.
} 
durement les moines gyrovagues catholiques locaux pour leur paresse, leur insistance à exiger la charité et leur tendance à piller les récoltes dans son traité De opere monachorum, écrit entre 405 et 406 . Il utilise pour les décrire une formulation proche de celle qu'il emploie pour les circoncellions donatistes ${ }^{26}$. Cependant, Augustin a réservé ce terme aux seuls donatistes, sans doute parce qu'il a essayé de lui donner un contenu péjoratif, les personnes rodant autours des celliers pour les piller. Or, le terme circoncellion a connu une prospérité inattendue. Brent Dwight Shaw a d'ailleurs récemment remarqué qu'à partir de Cassiodore au $\mathrm{VI}^{\mathrm{e}}$ siècle et jusqu'au $\mathrm{XII}^{\mathrm{e}}$ siècle, le terme circoncellion, détaché de toute allusion à l'hérésie donatiste, en est venu à désigner toute forme de monachisme itinérant ${ }^{27}$. Il faudrait admettre que Cassiodore et Isidore de Séville ont réalisé un contresens complet dans l'emploi de ce terme, seulement un siècle après la disparition des circoncellions donatistes. Le terme circoncellion désignerait soit des moines vagabonds suspectés de trop trainer autours des greniers, soit, selon William Frend, des ascètes itinérants tournant autours des cellae des martyrs. L'existence de circoncellions catholiques expliquerait que les ascètes itinérants donatistes aient préféré le terme, qui leur était propre, d'agonistici ou de confessores.

Peu d'attention a été portée aux passages de l'œuvre d'Augustin évoquant les trajectoires individuelles de personnes ayant rejoint les circoncellions. Dans une lettre écrite en 396, Augustin évoque un certain Primus, colon et sous-diacre de l'église de Spaniensis, qui se serait converti au donatisme en compagnie de deux nonnes habitant le fundus d'un propriétaire catholique. Ils seraient entrés dans une bande errante de circoncellions comptant dans ses rangs beaucoup de femmes qui refusaient le mariage et toute règle de vie pour se livrer à la boisson et à toutes sortes de dérèglements. Primus a été plus tard tué dans une action contre le domaine d'un propriétaire terrien catholique ${ }^{28}$. En 419, une moniale catholique, venue d'une bourgade étrangère et installée de sa propre initiative pour filler la laine sur le saltus Hispaniensis, correspondant sans doute à l'ecclesia Spaniensis, a été violée par le procurator de ce domaine. Ceci montre l'existence de formes de monachisme rural catholique en dehors de toute congrégation, totalement soumises à la domination du mâ̂tre ou de son intendant ${ }^{29}$. Augustin évoque aussi le cas d'une autre vierge, fille

\footnotetext{
${ }^{26}$ De opere monachorum, 21, 24; $28,36$.

${ }^{27}$ Cassiodore, En. in Ps., 132, 1; Isidore de Séville, De Ecclesiasticis Officiis, II, 16, 7-8. Ainsi Wibaldus abbé du monastère de Corvey au XII ${ }^{e}$ siècle, a dénoncé deux de ses moines vagabonds en tant que circoncellions (PL 189, col. 275). Pourtant, Brent D. Shaw disqualifie tous les auteurs ayant évoqué les circoncellions après le $\mathrm{V}^{*}$ siècle, alors que ceux-ci se sont souvent limités à reprendre en les déformant les travaux d'Augustin. Voir Brent Dwight SHAW, Who Were the Circumcellions?, dans: A. MERRILS (dir.), Vandals, Romans and Berbers, Ashgate 2004, p. 227-258.

${ }^{28}$ Ep. 35, 2; 108, 6, 19.

${ }^{29}$ Ep., $15^{*}, 3$.
} 
d'un colon de l'Église catholique, qui s'est convertie au donatisme contre l'avis de son père. Cette femme a sans doute rejoint les rangs des circoncellions ${ }^{30}$. Augustin évoque enfin dans une lettre de 411 ou 412 Donatus, ancien diacre et colon de l'Église catholique devenu donatiste et coupable du meurtre d'un prêtre catholique lorsqu'il était membre d'une bande de circoncellions ${ }^{31}$. Le modèle ascétique itinérant des circoncellions exerçait une forte attraction sur le clergé rural catholique, alors qu'à la même époque Augustin se plaignait de la difficulté de recruter des moines pour son monastère clos d'Hippone ${ }^{32}$. Il constituait une des seules façons de s'émanciper de la tutelle pesant du dominus, une grande partie de ce clergé rural étant soumis au statut du colonat. L'attrait des circoncellions explique qu'Augustin ait formé pour les concurrencer à partir de 396 un monastère pour les clercs d'Hippone et ait choisi au moins un évêque rural dans ce monastère ${ }^{33}$. Les circoncellions formaient donc une nouvelle élite rurale issue en moins en partie du colonat, caractérisée par sa liberté de mouvement et son ascétisme.

Il reste à préciser la question de la nature des circoncellions dans la première moitié du IV $\mathrm{V}^{\mathfrak{e}}$ siècle. Or, un témoignage méconnu d'un auteur gaulois du $\mathrm{V}^{\mathfrak{e}}$ siècle, Gennadius, montre, là encore, leur caractère monastique. Il rapporte avoir lu le traité $A d$ confessores et virgines, valorisant l'usage de la chasteté, d'un certain Macrobius, qui aurait été d'abord prêtre catholique avant de se convertir au donatisme et de devenir, comme Optat de Milev l'a affirmé, l'évêque donatiste de Rome vers $366^{34}$. Pour André Mandouze, à la suite de Paul Monceaux, Gennadius aurait confondu l'évêque donatiste de Rome avec l'auteur catholique du traité $A d$ confessores et virgines ${ }^{35}$. Ce traité a même été identifié par Harnack avec le traité De singularitate clericorum du pseudoCyprien. Néanmoins, cette identification a été repoussée à juste titre par JeanLouis Maier, car le traité de Macrobius était destiné aux confessores et non aux clercs. Or, le terme de confessores était strictement réservé par les catholiques après la persécution de Dioclétien aux personnes ayant connu le martyre et ne pouvait être utilisé pour des personnes vivantes. Justement, Optat et Au-

${ }^{30}$ Ep., $35,4$.

${ }^{31}$ Ep., 133; 139, 2.

32 Voir Charles MUNIER, Problèmes monastiques et conciles africains, dans: Augustinianum 39 (1999), p. 149-168.

${ }^{33}$ Sermones, 355; 356; Ep., 209; Ep., 20* (Antonius de Fussala).

${ }^{34}$ Optat, Adv. Parm., II, 4; Gennadius, De viris illustr., 5: Macrobius presbyter et ipse, ut ex scriptis Optati cognovi, donatianorum postea in urbe Roma occultus episcopus fuit. Scripsit, cum adhuc in ecclesia dei presbyter esset, Ad confessores et virgines librum moralis quidem sed valde necessariae doctrinae et praecipue ad custodiendum castitatem aptissimis valde sententiis communitum. Claruit inter nostros primum Africae et inter suos, id est donatianos sive montenses postea Romae. Voir Jean-Louis MAIER, Le dossier du donatisme, (Texte und Untersuchungen zur Geschichte der altchristlichen Literatur, 135), Berlin 1989, $\mathbf{n}^{\circ} 120$, t. II, p. 305.

${ }^{35}$ Cf. PCBE, I, Macrobius 1, p. 662. 
gustin, qui ont insisté sur la volonté de martyre des circoncellions tant au IV qu'au $\mathrm{V}^{\mathfrak{c}}$ siècle, ont remarqué que ceux-ci se dénommaient eux-mêmes agonistici ou confessores agonistici $i^{36}$. Gennadius aurait en fait attribué à la période catholique de Macrobius, pour ne pas être critiqué pour avoir lu un ouvrage hérétique, un traité que Macrobius aurait rédigé à l'usage des circoncellions. Macrobius a donc été une sorte de théoricien des pratiques ascétiques des circoncellions et des vierges ou sanctimoniales qui les accompagnaient. Ce prêtre catholique a sans doute rejoint les circoncellions donatistes, comme les diacres Primus et Donatus précédemment évoqués ${ }^{37}$. On attribue avec vraisemblance à Macrobius, évêque donatiste de Rome en 366, la passion des martyrs donatistes Isaac et Maximinianus de Carthage. Ils ont été exécutés en 347 par Paul et Macaire, les envoyés de l'empereur Constant qui a rétabli de force l'unité entre les deux Églises ${ }^{38}$. Cette passion introduit une louange des donatistes qui avaient en masse été exécutés par Paul et Macaire en Numidie peu de temps auparavant. Il s'agit d'une allusion claire au massacre de nombreux circoncellions tentant de protéger l'évêque schismatique Donatus de Bagaï au début de l'année 347. Macrobius, très favorable aux circoncellions contrairement à certains de ses collègues, a connu une surprenante promotion en parvenant à la tête de la communauté donatiste exilée à Rome. Dans une période de crise, marquée par la disparition d'une partie des évêques, les circoncellions ont structuré la résistance donatiste. Le terme de circoncellions, qui semble donc avoir eu un sens unique pendant toute la durée du schisme donatiste, a ainsi désigné uniquement des confréries de moines mendiants itinérants au $\mathrm{IV}^{\mathfrak{e}}$ et $\mathrm{V}^{\mathfrak{e}}$ siècle.

Il faut maintenant s'interroger sur l'ampleur et le sens des troubles fomentés par les circoncellions en Numidie tant au $I V^{e} q u ' a u V^{e}$ siècle. Selon Optat de Milev, les circoncellions, menés par deux chefs nommés Axido et Fasir se désignant par le terme de duces sanctorum, ont commis de nombreuses violences

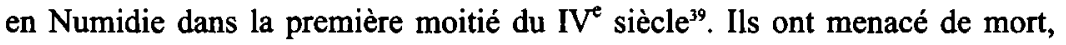
par le moyen de lettres, des propriétaires terriens s'ils ne supprimaient pas les dettes pesant sur certains de leurs dépendants. Ils ont forcé des notables à courir devant leurs voitures conduites par leurs propres esclaves, par un rite typique d'inversion sociale, avant d'être massacrés par le comte Taurinus. Augustin, dans une lettre datée de 417 , a rappelé ces crimes et en a évoqué quelques

${ }^{36}$ Optat, Adv. Parm., III, 4, 3-6; Augustin, En. in Ps., 132, 3.

${ }^{37}$ Voir n. 32.

${ }^{38}$ Cet auteur, qui se nomme Macrobius et se dit martyr, comme le faisait les circoncellions, affirme dans cette Passion avoir été forcé de partir d'Afrique et espérer y retourner bientôt. Il semble avoir été témoin du martyre d'Isaac et de Maximinianus entre 345 et 348 puis avoir été exilé. Cette identification a été en particulier défendue par P. Monceaux et J. L. Maier (Le dossier du donatisme [voir n.34], n⿳36, t. I, p. 256-260). Cf. Paul MONCEAUX, Histoire littéraire de l'Afrique chrétienne, t. V, Paris 1912, p. 156-161; PCBE, I, Macrobius 1, p. 662.

${ }^{39}$ Optat, Adv. Parm., III, 4, 3-6. 
autres commis à la même époque $\mathrm{e}^{40}$. Les circoncellions ont protégé des esclaves ayant pillé les celliers de leurs maîtres, ont forcé des notables à tourner des meules de boulangerie comme s'ils étaient des esclaves et ont mis en fuite collecteurs d'impôts et fonctionnaires. Possidius, dans sa biographie d'Augustin, souligne que les circoncellions, au mépris des lois, empêchaient certains $d o-$ mini d'exercer la plénitude de leurs droits de propriété sur leurs domaines. Cependant, seuls les propriétaires refusant d'obéir à leurs ordres subissaient leurs sévices $^{41}$. Ni Optat, ni Augustin, ni Possidius en effet n'évoquent un seul meurtre commis par les circoncellions, un seul pillage ou occupation de domaine dans la première moitié du IV $\mathrm{e}^{\mathrm{e}}$ siècle. Le terme de révolte sociale ou de jacquerie, utilisé par la majorité des commentateurs, semble impropre pour désigner l'instauration en Numidie d'une forme populaire de justice sociale établie par la force par les circoncellions. Il s'agirait en effet d'une bien curieuse révolte dans laquelle les chefs insurgés prévenaient leurs victimes par le moyen de lettres. D'autre part, la révolte est un trouble de courte durée, pendant lequel des éléments protestataires tentent de détruire les cadres sociaux existants. Au contraire, l'action des circoncellions semble s'être déroulée pendant une période relativement longue, peut-être dès les années 320 , et semble se limiter à imposer un code moral dans les rapports entre maîtres et dépendants agricoles ${ }^{42}$. La révolte africaine du temps du comte Taurinus est donc un mythe historiographique qui reprend à la lettre la condamnation polémique par Optat et Augustin des circoncellions comme latrones et comme seditiosit ${ }^{43}$.

Or, ce sont les évêques donatistes eux-mêmes qui ont demandé l'intervention violente du comte de l'armée d'Afrique Taurinus, parce qu'ils ne parvenaient pas à châtier les circoncellions par la simple discipline ecclésiastique ${ }^{44}$. Ceci peut s'expliquer si le statut privilégié de moine dont bénéficiaient les circoncellions les avait placés sous la seule juridiction épiscopale, limitant la liberté d'action des autorités civiles et militaires. Une loi d'Arcadius de 398 rend responsable les évêques des clerici, des synoditae et des monachi libérant certains condamnés jugés innocents, qui devaient être sévèrement punis ${ }^{45}$. Ceci peut expliquer la violence de la répression menée par Taurinus, condamné

\footnotetext{
${ }^{40}$ Augustin, Ep., 185, 4, 15.

${ }^{41}$ Possidius, Vita Aug., X.

${ }^{42}$ Taurinus a pu occuper le poste de comes rei militaris d'Afrique à tout moment entre 321 et 345. Les menées d'Axido et Fasir ne sont donc pas forcément à placer dans les années 340. Voir PLRE, I, Taurinus, p. 878; PCBE, I, Taurinus, p. 1217.

${ }^{43}$ Augustin, Ep., 105, 2, 5; 88, 8: Vivunt ut latrones, moriuntur ut circumcelliones, honorantur ut martyres; et tamen nec latrones aliquando audivimus eos quos depraedati sunt, excaecasse. Voir aussi C. PETIL., II, 23, 52; 83, 183; Sermo 53A, 13.

${ }^{44}$ Optat, Adv. Parm., III, 4, 6: homines in ecclesia corrigi non posse.

${ }^{45}$ CTh., LX, 40, 16.
} 
avant l'intervention des évêques donatistes à subir sans pouvoir réagir les insolences des circoncellions.

On ne peut séparer deux phases nettes dans les violences commises par les circoncellions, qui auraient été selon Emin Tengström d'abord liées à des préoccupations sociales puis uniquement à des questions religieuses. En effet, les circoncellions exerçaient encore en 401 , plus de cinquante ans après la répression de Taurinus, certaines formes de justice populaire dans les campagnes africaines. Comme l'a remarqué Jean-Pierre Brisson, Augustin, en 401 ou au début de 402, a en effet contesté le droit aux circoncellions de punir des homines mali, dans un passage d'un traité destiné aux catholiques, l'Epistula ad Catholicos. En effet, il affirme que les circoncellions, même lorsqu'ils s'en prenaient à des pessimi homines, n'avaient aucun droit d'usurper un pouvoir de justice hors du cadre du procès régulier ${ }^{46}$. Il s'agissait pour lui d'un latrocinium, un brigandage, les circoncellions inordinati usurpant la potestas des autorités légitimes ${ }^{47}$. De même, Optat de Milev a condamné les circoncellions pour avoir établi leur iudicium et leur imperium sur une partie de la Numidie ${ }^{48}$. Ainsi, il existait encore en $\mathbf{4 0 1}$ des catholiques qui approuvaient l'action sociale des circoncellions donatistes, qu'ils auraient dû déconsidérer en tant que schismatiques et séditieux. Or, Augustin, nous l'avons vu, a critiqué dans ce même sermon immédiatement auparavant le titre de moine que se donnaient les circoncellions, ce qui montre que les circoncellions se prévalait de cette qualité pour légitimer leur action ${ }^{49}$.

Certaines formes de justice populaire ont été tolérées par les autorités romaines depuis l'époque républicaine, en les limitant aux populations urbaines. On en connaît un exemple pour l'époque d'Augustin, qui peut être rapproché de l'action des circoncellions. Augustin s'est senti obligé, à l'occasion d'une émeute urbaine à Hippone marquée par le lynchage d'un douanier entre 409 et 412 , d'expliquer à ses fidèles catholiques qu'ils ne pouvaient eux-mêmes se faire justice sur un fonctionnaire corrompu ${ }^{50}$. Augustin a dû répondre dans ce sermon aux critiques du peuple contre l'inefficacité de son intercession auprès des autorités pour mettre fin aux abus de ce militaire. Le jugement populaire

\footnotetext{
${ }^{46}$ Ep. ad Cath., XX, 54. Cf. Jean-Paul BRISSON, Autonomisme et christianisme (voir n. 3), p. 333.

${ }_{47}$ Ibid., XX, 53: Culpantur etiam qui prohibent a malo, si modum peccati modus cohercitionis excedat. Item iure culpandi sunt, qui turbide atque inorditate in eos cohercendos insiliunt, qui nulla sibi lege subiecti sunt. Ces masses anarchiques ne peuvent être que les circoncellions, évoqués explicitement dans le paragraphe suivant.

${ }^{48}$ Optat, Adv. Parm., III, 4, 5.

${ }^{49}$ Ep. ad Cath., XVI, 40.

${ }^{50}$ Sur cette émeute, connue par le Sermon 302 et le post sermonem Morin Guelf., 25, voir Claude LEPELLEY, Le patronat ecclésiastique aux $\mathrm{IV}^{\boldsymbol{t}}$ et $\mathrm{V}^{\boldsymbol{e}}$ siècles: continuités et ruptures avec le patronat classique, dans: Eric REBILLARD et Claire SOTINEL (dir.), L'évêque et la cité du IV ${ }^{e}$ siècle au $V^{e}$ siècle. Image et autorité (CEFR 248), Rome 1998, p. 17-33.
} 
était donc perçu comme un palliatif à l'impuissance des évêques ${ }^{51}$. Augustin reconnaissait une certaine légitimité à des formes de justice populaire même si elles pouvaient être assimilées à des latrocinia ${ }^{52}$. Or, fait particulièrement étonnant, le droit à une justice populaire était parfois explicitement reconnu par les autorités. Le proconsul d'Afrique Seranus en 397 a chargé les habitants donatistes d'Abitina de confisquer la basilique de la cité voisine de Membressa après leur occupation par un évêque donatiste s'étant séparé de ses collègues, Salvius ${ }^{53}$. Il a été humilié devant la population de Membressa, étant forcé de danser avec des cadavres de chiens accrochés à son $\operatorname{cou}^{54}$. Seranus a accepté ces formes de violences symboliques parfaitement prévisibles, comme s'il était légitime d'humilier publiquement un évêque schismatique ${ }^{55}$. Les circoncellions ont comblé un vide en exerçant des formes de justice populaire en milieu rural.

Les circoncellions, se considérant comme des moines, ont pu légitiment se sentir investis de la mission de mettre fin à des injustices sociales dénoncées régulièrement par les pères de l'Église, dont ils se différenciaient seulement par la radicalité de la solution proposée, l'usage de la force. Il est possible que les circoncellions n'aient supprimé que les seuls prêts à taux usuraires, dénoncés par exemple par Grégoire de Nazianze et Ambroise ${ }^{56}$. D'autres moines gyrovagues, en Orient, avaient des pratiques proches des circoncellions. Ainsi Syméon Stylite dans une lettre apparemment authentique adressée à un village de Syrie au $\mathrm{V}^{\mathrm{e}}$ siècle a imposé aux notables de celui-ci de ne pas dépasser un taux de crédit de $0,5 \%$ par an sous peine d'excommunication ${ }^{57}$. Eustathe de Sébastée et les moines qui l'entouraient ont été condamnés au concile de Gangres entre 340 et 343 car ils encourageaient des esclaves à désobeir à leurs maîtres ou à fuir et réclamaient être les seuls bénéficiaires de l'aumône ${ }^{58}$.

Les circoncellions se sont sans doute limités à protéger les personnes devenues esclaves depuis peu, principalement pour dettes, un problème fréquent en

\footnotetext{
${ }^{51}$ Sermo 302, 17.

${ }^{52}$ Sermo 302, 21.

${ }^{53}$ C. Ep. Parm., III, 6, 29.

${ }^{54}$ Augustin, C. Ep. Parm., III, 6, 19 (PCBE, I, Salvius 2, p. 1025-1026).

${ }^{55}$ Ces violences peuvent être comparées à celles pratiquées en France lors des guerres de religion. Voir Natalie Zemon DAVIS, Les rites de violence, dans: Les cultures du peuple. Rituels, savoirs et résistance au XVI siècle, Paris 1979, p. 251-307.

${ }_{56}$ Saint Ambroise, De Nabaoth, 21-24; Grégoire de Nazianze, Or., XVI, 18-19.

${ }^{57}$ Hermann LIETZMANN, Das Leben des Heiligen Symeon Stylites, Leipzig 1908 (Texte und Untersuchungen zur Geschichte der altchristlichen Literatur 32), p. 184-188. Alexandre l'Acémète, meneur d'un groupe de moines errants, a maudit à la fin du IV' siècle certains notables de Palmyre qui refusaient de supprimer les dettes de personnes pauvres (Vie d'Alexandre, 19; 35).

${ }^{58}$ Concile Gangres, canon 3; Sozomène, H. E., III, 14, 31. Ce concile n'est pas daté avec précision. Cf. Jean GRIBOMONT, Le monachisme au IV siècle en Asie Mineure: de Gangres au Messalianisme, dans: Studia Patristica 2 (1957), p. 400-415.
} 
Afrique à cette époque. En outre, comme l'indique Augustin, les circoncellions devaient offrir une sorte de droit d'asile ecclésiastique ainsi que leur patrocinium à des esclaves, sans doute maltraités ${ }^{59}$. Augustin a défendu des personnes qu'un propriétaire terrien réclamait illégalement comme esclaves car leur père, de naissance libre, était devenu actor de celui-ci ${ }^{60}$. Or, Augustin a lui-même justifié le recours à la violence pour libérer des personnes libres injustement réduites en esclavage, un usage qu'il condamnait sévèrement lorsqu'il était exercé par les circoncellions. En 428, il a défendu les fidèles catholiques de l'Église d'Hippone qui avaient libéré par la force cent vingt personnes réduites à la servitude après avoir été illégalement vendues par leurs parents ou enlevées par des bandits ${ }^{61}$. Les circoncellions pouvaient même s'appuyer sur la législation impériale, dans le contexte d'une crise agraire endémique en Afrique du Nord au début du IV $\mathrm{IV}^{\mathrm{e}}$ siècle. Une constitution de Constantin de 322, adressée à Ménandre, vicaire ou comte d'Afrique, accorde la possibilité aux Africains ne pouvant nourrir leur famille de bénéficier de distributions d'aliments et de vêtements par le Fisc. Constantin a significativement motivé sa décision par le souci d'éviter que les pauvres, egentes, soient obligés de vendre leurs enfants comme esclaves ou de commettre des actes criminels $^{62}$. L'appel des paysans aux circoncellions évoque les pétitions envoyées aux empereurs par des communautés rurales africaines au Haut Empire, par exemple celle du Saltus Burunitanus, dans laquelle des conductores de domaines impériaux sont accusés de battre illégalement de verges des paysans $^{63}$. Les lettres des circoncellions peuvent être rapprochées des rescrits impériaux imposant aux conductores et régisseurs de cesser leurs mauvais traitements. Les circoncellions tentaient ainsi de se substituer aux empereurs comme patron des populations rurales.

Cependant, les circoncellions ont principalement usurpé le patronage exercé par les évêques donatistes sur les populations rurales et leur prérogative de la pénitence grâce au prestige religieux dont ils disposaient en tant que moines. Ceci explique que ce soit les évêques donatistes eux mêmes qui aient demandé l'intervention du comte Taurinus au début du $\mathrm{V}^{\mathfrak{e}}$ siècle. Augustin a fréquemment intercédé en faveur de paysans qui lui envoyaient des requêtes pour des

${ }^{59}$ Augustin utilise la formule Ad illorum patrocinium confugisset (Ep., 185, 4, 15) qu'il faut comparer avec le titre du chapitre du Code Théodosien consacré au droit d'asile dans les églises: De his qui ad ecclesias confugisset (CTh., IX, 45). Une loi de 398 interdit justement le droit d'asile pour des esclaves (CTh., LX, 45, 3).

${ }^{60}$ Augustin, Ep., 24*.

${ }^{61}$ Augustin, Ep., 10*, 7. Une loi de Constantin de 315 dédiée au vicaire d'Afrique évoque l'existence en Afrique de bandes organisées spécialisées dans les enlèvements d'enfants (CTh., LX, 18, 1 = C. J., LX, 20, 16 en 315)

${ }^{62} \mathrm{CTh} ., \mathrm{XI}, 27,2$.

${ }^{63}$ Cette pétition sur inscription, adressée à Commode, est datée de 165 (CIL VIII, 10570). 
questions de dettes ou de régisseur tyrannique ${ }^{64}$. L'ascèse pratiquée par les circoncellions ainsi que leur vocation au martyre leur a donné l'autorité suffisante pour imposer leur morale puritaine à la société rurale de Numidie. Leurs violences peuvent être interprétées comme des formes extrêmes de pénitence. En effet, selon Optat, il aurait été possible aux propriétaires terriens d'éviter ces violences en s'humiliant et en suppliant, c'est-à-dire en adoptant la posture du pénitent ${ }^{65}$. Ceci évoque les pénitences très rigoureuses des fidèles africains au III ${ }^{\mathrm{e}}$ siècle selon Tertullien. Le pécheur revêtu d'un sac de toile et de cendres devait ramper devant les frères et lécher leurs genoux ${ }^{66}$. Optat a particulièrement critiqué l'obsession des donatistes pour la pénitence, qu'ils auraient même imposée à de petits enfants, à des femmes mariées et à des vierges qui auraient été brisés et persécutés par ces pratiques ${ }^{67}$. Possidius, dans un passage souvent négligé, affirme que les circoncellions s'en prenaient d'abord à leurs proches, et ensuite seulement à des étrangers ${ }^{68}$. On peut donc suspecter une entreprise de moralisation de l'ensemble de la société rurale de la Numidie, commençant par les paysans eux-mêmes, notamment en matière de morale sexuelle, pour s'étendre ensuite aux propriétaires terriens et aux questions sociales, dans le contexte d'une société fortement christianisée. Or, Augustin ne refusait pas lui-même l'idée de formes violentes de pénitence. Entre 423 et 429, il a défendu des clercs de Thagaste qui avaient frappé un jeune curiale ayant enlevé une moniale pour satisfaire son goût pour la débauche ${ }^{69}$. La bastonnade semble avoir été un mode de pénitence régulièrement imposé par des clercs en Afrique au $I V^{\mathfrak{e}}$ et $\mathrm{V}^{\mathfrak{e}}$ siècles, même si les conciles et la législation impériale ont toujours combattu cette pratique ${ }^{70}$. Les circoncellions n'ont pas hésité à appeler verus Israheles le bâton qu'ils portaient habituellement, comme s'il avait pour fonction de contribuer à créer un peuple véritablement chrétien $^{71}$. Libanios a critiqué les moines de Syrie qui pillaient les domaines ruraux des païens et leurs sanctuaires. Il affirmait d'ailleurs que ces moines se donnaient pour nom sophronistai, les modérateurs, comme s'ils s'assimilaient

\footnotetext{
${ }^{64}$ Il est intervenu notamment en faveur d'un groupe de colons obligés par le régisseur de leur dominus à verser deux fois les redevances prévues. Protégeant en 397 un paysan débiteur auquel il avait donné asile, Augustin a lui-même remboursé ses créanciers (Ep., 247; 268).

${ }^{65}$ Optat, Adv. Parm., III, 4, 4: metu mortis humiles impellentur in preces.

${ }^{66}$ Tertullien, De pudicitia, 13.

${ }^{67}$ Optat, Adv. Parm., II, 26, 1-3.

${ }^{68}$ Possidius, Vita Aug., X: nec suis, nec alienis aliquando parcebant.

${ }^{69}$ Ep., 9*, 1-3.

${ }^{70}$ Voir Ep., 8*, 2; Lisa DOSSEY, Judicial Violence and the Ecclesiastical Courts in Late Antique North Africa, dans: Ralph MATHISEN (dir.), Law, Society and Authority in Late Antiquity, Oxford 2001, p. 98-114.

${ }^{71}$ Ps. contra Partem Donati, 157-162.
} 
à une véritable police nurale ${ }^{72}$. Les circoncellions, qui ont d'ailleurs lutté contre les cultes païens, ont pu s'attribuer la même fonction.

De plus, les circoncellions pouvaient revendiquer une autorité ecclésiastique supérieure à celle des évêques par leur aspiration au martyre. Ils étaient en effet nostalgiques de l'époque des persécutions païennes. Ils ont mené des attaques suicidaires contre des itvenes païens lors des spectacles que ceux-ci organisaient dans la première moitié du IV $V^{e}$ siècle $^{73}$. Chacune des phases de répression, au temps du comte Taurinus, après les deux interdictions du donatisme en 347 puis après 405 , a été suivie par une vague massive de martyre volontaire parmi les circoncellions. Théodoret de Cyr a remarqué que c'est cette aspiration au martyre qui justifiait les fortes aumônes qu'ils recevaient ${ }^{74}$. La prétention de ces confessores agonistici, comme ils se nommaient, à usurper le pouvoir des évêques donatistes s'explique par l'autorité acquise lors des persécutions du III ${ }^{\mathrm{e}}$ siècle par les confessores, les chrétiens arrêtés par les païens qui n'avaient pas été exécutés ${ }^{75}$. Saint Cyprien a dû lutter contre l'usurpation par certains confessores de prérogatives épiscopales, comme la possibilité d'accorder le pardon aux lapsi, les chrétiens qui avaient sacrifié ${ }^{76}$. Une hypothèse séduisante, bien que totalement invérifiable, verrait en Axido et Fasir, les deux chefs des circoncellions ou confessores agonistici du début du $\mathrm{IV}^{\mathrm{e}}$ siècle se nommant duces sanctorum, deux confesseurs ayant été libérés à la fin de la persécution de Dioclétien en 305. Ceci permettrait de comprendre tant leur autorité morale que le modèle du martyre qu'ils proposaient aux fidèles rigoristes qui les accompagnaient. La sanctification des circoncellions par certains donatistes constituait bien évidemment une forme enviable de promotion sociale post mortem. Tous les textes donatistes insistent sur l'autorité des martyrs face à des évêques faibles ayant souvent sacrifié aux dieux païens. Les évêques donatistes n'avaient pas prévu que cette présentation conduirait à miner leur propre autorité. Il est du reste remarquable que la seule Passion connue d'un évêque donatiste, Marculus de Vegesala en 347, ne mentionne absolument pas le martyre de nombreux circoncellions peu de temps auparavant ${ }^{77}$.

L'autorité acquise par les circoncellions s'explique aussi parce qu'ils pouvaient se poser en recours contre l'impuissance des évêques ruraux envers les

\footnotetext{
${ }^{72}$ Libanios, Or., XXX, 12.

${ }^{73}$ Sur ce sujet, voir Claude LEPELLEY, Iuvenes et Circoncellions. Les demiers sacrifices humains de l'Afrique antique, dans: Antiquités africaines 15 (1980), p. 261-273.

${ }^{74}$ Théodoret de Cyr, Haereticarum fabularum compendium, IV, 6.

${ }^{75}$ Optat, Adv. Parm., III, 4, 3-6; Augustin, En. in Ps., 132, 3.

${ }^{76}$ Cyprien, Ep., 27, 1. Cyprien insiste sur le fait que les confessores ne pouvaient se séparer de l'Église en refusant l'autorité des évêques (De lapsis, 6; Ep., 54, 3).

${ }^{77} \mathrm{Ce}$ modèle de l'évêque donatiste s'oppose aux circoncellions et aux évêques qui les soutenaient, comme Macrobius, évêque de Rome en 366, auteur du traité Ad confessores et virgines et d'une Passion d'Isaac et de Maximianus qui contient, nous l'avons vu, des allusions explicites aux circoncellions.
} 
potentes. La particularité de l'Église africaine était de compter un très grand nombre de petits évêchés se limitant à un village ou même à un domaine foncier particulier. Des domaines fonciers privés ou impériaux de très grandes dimensions se partageaient en effet le territoire de la Numidie ${ }^{78}$. Ces évêques étaient d'origine humble et dépendants du propriétaire terrien qui avait suscité leur nomination. Antonius, évêque du petit castellum de Fussala recueilli dans le monastère d'Augustin parce qu'il était pauvre, s'est plaint d'avoir été persécuté par le sénateur Celer qui y possédait de grandes propriétés ${ }^{79}$. En 411 , le tribun Marcellinus a même imposé aux intendants des domaines et aux anciens (seniores) des villages de convoquer tous les évêques catholiques et donatistes pour la conférence de réconciliation de Carthage ${ }^{80}$. Les évêques donatistes devaient supprimer le risque de formation d'une hiérarchie religieuse parallèle au sein du donatisme. Ils ont ainsi interdit au clergé rural donatiste, après la répression menée par le comte Taurinus, d'ensevelir des circoncellions dans leurs églises, surtout s'ils avaient commis un suicide volontaire ${ }^{81}$. Les diacres et prêtres souvent anciens colons qui en faisaient partie se libéraient de l'emprise de leur dominus et de leur évêque et pouvaient concurrencer les évêques au nom de leur ascétisme ${ }^{82}$. Les circoncellions formaient donc une nouvelle élite rurale. En outre, les circoncellions, qualifiés de irvenes par Augustin, pouvaient s'opposer aux seniores des villages ${ }^{83}$. La constitution de groupes de circoncellions substituait des réseaux de sociabilité horizontaux à ceux verticaux mis en place par les élites. Augustin rapporte, nous l'avons vu, le martyre volontaire de circoncellions se jetant sans armes contre des iuvenes païens lors des combats simulés dans les amphithêâtres qu'ils organisaient. Les circoncellions, se définissant comme agonistici, ont pu s'inspirer du modèle agonistique des iuvenes, qu'ils auraient inversé et détourné en occasions supplémentaires de martyre. Les circoncellions ruraux se définissaient ainsi comme égaux par leur ascétisme et leur courage aux fils de notables municipaux païens participant aux organisations de iuvenes à la tête de leurs dépendants rurau ${ }^{84}$. Ce-

${ }^{78}$ Voir Serge LANCEL, Études sur la Numidie d'Hippone au temps de saint Augustin. Recherches de topographie ecclésiastique, dans: MEFRA 96 (1984), p. 1085-1113; François JACQUES, Propriétés impériales et cités en Numidie méridionale, dans: Cahiers du Centre Glotz 3 (1992), p. 123-139.

${ }^{79}$ Augustin, Ep., 139, 2; 209, 5.

${ }^{80}$ Gesta, I, 5, 1. 37.

${ }^{81}$ Optat, Adv. Parm., III, 4, 7. Cette interdiction a été tournée par un prêtre de Subbula.

${ }^{82}$ Augustin évoque deux diacres catholiques et colons, Primus vers 396 et Donatus vers 411 , bien entendu indignes, passés aux circoncellions (Ep., 133; 139, 2; Ep., 108, 6, 19). Voir n. 30-33.

${ }^{83}$ C. Ep. Parm., I, 11, 17.

${ }^{84}$ François Jacques a montré que le déclenchement de la révolte africaine de 238 contre l'empereur Maximin le Thrace a impliqué non seulement des iuvenes membres des élites urbaines de Thysdrus mais aussi des itvenes de statut plus humble et d'origine paysanne. Cf. 
pendant, les circoncellions étaient itinérants, à la différence des iuvenes païens. La résidence fixe dans un village les auraient en effet placés en conflit direct avec les autorités traditionnelles, régisseurs, évêques ou seniores.

À partir de l'interdiction du donatisme en 405 , renouvelée en 411 , les circoncellions se sont substitués aux évêques en fuite en structurant la résistance des donatistes refusant de se convertir au catholicisme. On peut observer une rupture dès la période 403-405, marquée par les premières agressions de circoncellions contre des prêtres catholiques. Ces violences, dont Augustin donne quatre exemples, ont été habilement exploitées par les catholiques et ont été déterminantes pour la condamnation du donatisme en $405^{85}$. Les donatistes ont ainsi été accusés non seulement d'être des hérétiques mais aussi de mener des seditiones contre le pouvoir impérial ${ }^{86}$. Les évêques catholiques, en revendiquant des basiliques possédées par les donatistes et en exerçant leur prédication dans des zones rurales tenues par ceux-ci, semblent avoir délibérément cherché à créer un climat de tension leur permettant par la suite de condamner les violences des circoncellions. Les évêques catholiques, lors des conciles de Carthage de juin 403 et de juin 404, ont alors pu demander par deux fois la protection par les autorités civiles des communautés catholiques rurales contre les circoncellions ${ }^{87}$.

À partir de 405, les violences des circoncellions se sont principalement exercées contre les prêtres donatistes convertis au catholicisme. En fait, Augustin a répété inlassablement dans ses écrits un petit nombre d'agressions graves pour donner l'impression d'une violence omniprésente. Seulement quatre cas d'agressions liés aux circoncellions, dont une concernant un évêque, sont connus $^{88}$. Toutes les violences donatistes ne peuvent être attribuées aux cir-

François JACQUES, Humbles et notables. La place des humiliores dans les collèges de jeunes et leur rôle dans la révolte africaine de 238, dans: Antiquités africaines, 15 (1980), p. 217-230.

${ }^{85}$ Augustin a échappé de peu à une embuscade tendue par les circoncellions (Saint Augustin, Enchiridon, 5, 17; Possidius, Vita Aug., XII, 1-2; Sermon Mayence 62). Servus Dei, évêque de Thubursicum Bure, a été brutalisé en 404 par des circoncellions après avoir réclamé un domaine qu'ils occupaient (C. Cresc., III, 43, 47; Ep., 88, 7). Maximinianus, évêque de Bagaii, ayant obtenu en 404 la restitution d'une basilique, a été frappé dans son église par des circoncellions utilisant des gourdins et les débris d'un autel (C. Cresc., III, 43, 47; Ep., 88, 7; 185, 5, 27). Le sous-diacre Marcianus d'Urga, autre prêtre donatiste converti au catholicisme avant 405 , a été battu et lapidé par des clercs donatistes et des circoncellions (Ep. 105, 2, 3).

${ }^{86} \mathrm{CTh}$., XVI, 5, 38 (12 février 405 ).

${ }^{87}$ Gesta, III, 174, SC 224; Reg. Eccl. Carthag. Excerpta, l. 1028-1039.

${ }^{88}$ Restitutus, un prêtre donatiste converti au catholicisme officiant sur le domaine de Victorianensis a été en 405 battu en public et conduit en procession habillé d'une natte de jonc par des clercs donatistes et des circoncellions (C. Cresc., III, 48, 53; Ep. 88, 6; Ep. 105, 3; Possidius, Vita Aug., XII, 4). En 411 ou au début de 412, un prêtre donatiste devenu catholique, un autre Restitutus, a été tué par les circoncellions alors qu'un autre, Innocentius, a perdu un doigt et un ceil suite à un jet d'acide (Ep., 133, 1; 134, 2). Entre 412 et 418, Rogatus, évêque donatiste d'Assuras converti au catholicisme, a eu la langue et une main tranchée par les 
concellions, comme Augustin a tenté de le faire. Ceci explique qu'Augustin ait demandé au tribun Marcellinus à la fin de l'année 411 ou au début de l'année 412 de conduire de nombreux procès contre les circoncellions en leur donnant un maximum de publicité. Leurs crimes supposés et leur folie ne devaient pas faire l'unanimite ${ }^{89}$. Ces violences symboliques marquaient cependant l'exclusion définitive de traîtres des communautés rurales. Les circoncellions ont donc continué à faire la chasse aux mali homines, comme l'indiquait l'Epistula ad Catholicos d'Augustin de 401, passant simplement des propriétaires terriens tyranniques et des hommes de mauvaise moralité aux prêtres parjures.

L'enjeu principal de la période $405-418$ a été le contrôle des prêtres ruraux et des masses paysannes. La structure de la propriété foncière autour d'Hippone, monopolisée par un petit nombre de sénateurs et de curiales, explique que la plupart des prêtres et évêques ruraux aient été sous la responsabilité directe de leur dominus. La conversion forcée de ces prêtres sur l'ordre de leur maître a signifié l'impossibilité pour les paysans de pouvoir participer à un culte donatiste. Les paysans étaient ainsi l'objet des pressions conjuguées des propriétaires terriens donatistes et catholiques, ou de leurs intendants, n'hésitant pas à les convertir de force ${ }^{90}$. Honorius a d'ailleurs imposé, sous peine d'amende, aux domini catholiques de convertir leurs coloni donatistes au moyen de bastonnades répétées ${ }^{91}$.

Dans cette période troublée, les circoncellions, qui comprennent de nombreux membres du clergé, semblent s'être substitués aux évêques et aux prêtres donatistes soit en fuite, soit ayant cédé aux pressions en se convertissant au catholicisme ${ }^{92}$. Ainsi, l'évêque donatiste Macrobius d'Hippone s'est dissimulé dans la campagne au sein de groupes de circoncellions à partir de 405 , avant d'investir Hippone avec ces derniers en 408. Néanmoins, saint Augustin évoque le refus des circoncellions, parlant punique, de se soumettre à l'autorité de cet évêque cultivé qui avait des difficultés à les comprendre ${ }^{93}$. Plus tard, en 411 , Celer, sénateur donatiste converti au catholicisme après 405 , a confié à son procurator Spondius la mission d'empêcher, sans doute par la force, Ma-

circoncellions, des formes de violences hautement symboliques (Gesta cum Emerito, 9; Ep., $185,7,30$ ).

${ }^{89}$ Ep., 139, 1.

${ }^{90}$ Près de 48 paysans ont été rebaptisés sous la contrainte par un évêque donatiste vers 409 ayant pris en location un domaine impérial (Ep., 111, 1). P. Brown a bien montré comment Augustin en est venu, à partir de 405, à défendre la nécessité de la coercition en matière de religion. Voir Peter BRown, Saint Augustine's Attitude to Religious Coercion dans: Religion and Society in the Age of Saint Augustine, Londres 1972, p. 260-278.

${ }^{91}$ CTh., XVI, 5, 52.

${ }^{92}$ Une constitution d'Honorius de décembre 408 impose aux clercs donatistes l'amende de dix livres d'or frappant tous les hérétiques (CTh., XVI, 5, 39).

${ }_{93}$ Ep., 108, 6, 19;139, 2; 144. Augustin a dénoncé l'utilisation des circoncellions par les évêques et les prêtres donatistes (En in Ps., 10,5). 
crobius d'Hippone et les circoncellions qui l'accompagnaient de rouvrir les églises rurales donatistes ${ }^{94}$. Ces conflits ont entrainé la constitution de milices rurales. Antonius, placé par Augustin à la tête du petit évêché rural de Fussala, a terrorisé ses habitants avec l'aide d'une milice commandé par un ancien militaire ${ }^{95}$. Les troubles sociaux liés à la suppression du donatisme semblent beaucoup plus graves que ceux ayant frappé la Numidie dans la première moitié du $\mathrm{IV}^{\mathrm{e}}$ siècle. Augustin, dans une lettre adressée à l'évêque donatiste Macrobius en 409 ou en 410 , rapporte que les circoncellions soulevaient les paysans contre leurs maîtres et incitaient les esclaves à fuir, à piller les domaines de leurs maîtres et à les agresser ${ }^{96}$. Ces esclaves étaient sans doute des donatistes soumis à des maîtres catholiques. Inversement, Honorius a permis à l'esclave d'un maître donatiste de se réfugier auprès d'un évêque catholique, n'hésitant pas à remettre en cause le caractère intangible des relations entre maîtres et esclaves ${ }^{97}$.

Les circoncellions ont ainsi constitué une tentative des paysans de Numidie de créer une hiérarchie religieuse issue de leurs rangs, définie par son ascétisme, parallèle et concurrente à celle créée par les prêtres et les évêques donatistes. Les circoncellions constituaient un essai de formation d'une élite rurale autonome, même si elle était itinérante, face à un clergé et à des notables villageois inféodés aux grands propriétaires terriens et à leurs intendants. Les évêques donatistes, majoritairement issus de l'élite sociale comme l'a montré Claude Lepelley, se sont souvent opposés aux circoncellions qui usurpaient leurs fonctions, pénitence et patronage des paysans ${ }^{98}$. L'existence des circoncellions faisait courir à l'Église donatiste le danger d'un schisme interne. L'évêque donatiste Pétilien a exalté son Église comme celle des justes et des pauvres, par opposition à l'Église catholique. Augustin l'a accusé de vouloir rendre tous les hommes mendiants et ignorants, errants et querelleurs ${ }^{99}$. Cette critique méprisante des pauvres, très étonnante chez un père de l'Église, ne s'explique que si Augustin a voulu dans ce passage montrer le danger pour les donatistes de mettre en avant ce type de discours chrétien, en soi parfaitement banal, mais qui pouvait servir de justification aux excès des circoncellions, clairement visés par ce passage. Augustin se moquait ainsi des prétentions à la pauvreté de l'Église menée par le vir clarissimus Pétilien, qui risquait de se retrouver seul entouré des circoncellions et de la partie du clergé qui les soutenaient. En 373, des évêques rassemblés autours d'un certain Rogatus se sont

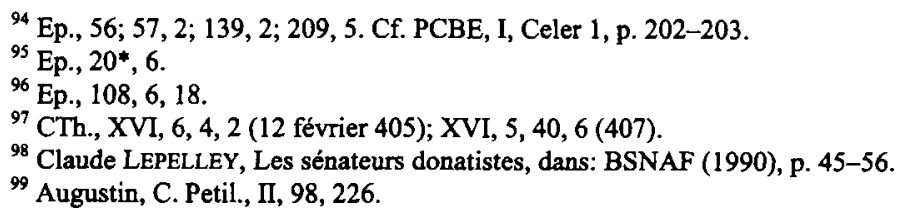


